\title{
MCEQ - numerical code for inclusive lepton flux calculations
}

\author{
Anatoli Fedynitch \\ Karlsruher Institut für Technologie, Institut für Kernphysik, Postfach 3640, 76021 Karlsruhe, \\ Germany \\ CERN EN-STI-EET, CH-1211 Geneva 23, Switzerland \\ E-mail: anatoli.fedynitch@cern.ch
}

\section{Ralph Engel*}

Karlsruher Institut für Technologie, Institut für Kernphysik, Postfach 3640, 76021 Karlsruhe, Germany

\section{Thomas K. Gaisser}

Bartol Research Institute, Department of Physics and Astronomy, University of Delaware, Newark, DE 19716, USA

\section{Felix Riehn}

Karlsruher Institut für Technologie, Institut für Kernphysik, Postfach 3640, 76021 Karlsruhe, Germany

\section{Todor Stanev}

Bartol Research Institute, Department of Physics and Astronomy, University of Delaware, Newark, DE 19716, USA

Calculations of atmospheric lepton spectra at very high energy experience a revived interest after the detection of the diffuse astrophysical neutrino flux by the IceCube observatory. In order to determine its spectral index and normalization with higher accuracy, the uncertainties of the atmospheric background have to modelled correctly and taken into account in the experimental fits. The available schemes, like a semi-analytic approach or Monte Carlo methods suffer either from a lack of detail or use too much CPU time and become inefficient. We have developed a numerical code based for the full system of coupled cascade equations which is shaped towards an efficient calculation of fluxes and achieves very high precision, comparable with Monte Carlo methods. Special attention lies on predictions of the prompt lepton flux using interchangeable physical parametrizations. Other features include detailed insight into hadrons participating in the cascade, the atmospheric density profiles and no restrictions on the definition of the primary cosmic ray flux.

The 34th International Cosmic Ray Conference,

30 July- 6 August, 2015

The Hague, The Netherlands

\footnotetext{
*Speaker.
} 


\section{Atmospheric cascades}

Hadronic interactions of high energy cosmic rays with air nuclei produce extensive cascades of secondary particles. The electromagnetic component of electrons and $\gamma$-rays dissipates its energy in form of bremsstrahlung and pair production. Hadrons can either interact, producing a sub-cascade, similar to the original one but at lower energy, or decay into other hadrons or leptons. Since leptons interact very weakly with the atmosphere, they reflect the history of the air-shower development and easily reach down to the surface. Volumetric or planar detectors at the surface measure the remaining particles, which have not decayed in the thermosphere, and attempt to reconstruct either the single air-shower as an exclusive state or, by integrating particle counts in time, to measure an inclusive physical observable. A typical observable is the differential inclusive flux $\Phi$, measured in units of $\left(\mathrm{cm}^{2} \mathrm{~s} \mathrm{sr} \mathrm{GeV}\right)^{-1}$. Aiming for the calculation of $\Phi$, our program solves the system of coupled cascade equations

$$
\begin{aligned}
\frac{\mathrm{d} \Phi_{h}(E, X)}{\mathrm{d} X}= & -\frac{\Phi_{h}(E, X)}{\lambda_{i n t, h}^{h}(E)}-\frac{\Phi_{h}(E, X)}{\lambda_{\text {dec }, h}(E, X)}-\frac{\partial}{\partial E}\left(\mu(E) \Phi_{h}(E, X)\right)+ \\
& +\sum_{l} \int_{E}^{\infty} d E_{l} c_{l\left(E_{l}\right) \rightarrow h(E)} \frac{\Phi_{l}\left(E_{l}, X\right)}{\lambda_{\text {int }, l}\left(E_{l}\right)}+\sum_{l} \int_{E}^{\infty} d E_{l} d_{l\left(E_{l}\right) \rightarrow h(E)} \frac{\Phi_{l}\left(E_{l}, X\right)}{\lambda_{\text {dec }, l}\left(E_{l}, X\right)},
\end{aligned}
$$

where the index $h=\pi^{+}, \pi^{-}, K^{+}, \ldots$ stands for one particle type. Therefore, each particle has its own cascade equation, representing the evolution of the energy spectrum as a function of atmospheric depth $X$. The factors $\lambda_{i n t, E}^{h}=m_{\text {air }} / \sigma_{p-\text { air }}^{\text {inel }}(E)$ are interaction lengths of $h$ in air. They vary slowly with energy only due to the inelastic (or particle production) cross-section and it takes comparable values for different particle species $h$, as far as measurements exist. In contrast, the decay length $\lambda_{d e c, E}^{h}(X)=c \tau_{h} E \rho_{\text {air }}(X) / m_{h}$ can vary by orders of magnitude, first due to the proportionality to the energy and second due to the particle life-time $\tau_{h}$. For heavy-flavor hadrons, such as $D$-mesons or $\Lambda_{C}$-baryons, the life-time is five orders of magnitude smaller compared to charged pions. At high energies the short life-time is compensated by $E$, therefore each particle has a lowenergy or decay dominated regime and a high-energy, interaction dominated regime. The energy at which this transition occurs is called critical energy $\varepsilon$ and takes values of $115 \mathrm{GeV}$ for $\pi^{ \pm}, 850$ $\mathrm{GeV}$ for $K^{ \pm}$and of several PeV for charmed mesons. Typically this feature is used to construct two limits in which the cascade equations can be solved analytically [1,2].

In the case of zero particle couplings $c_{l\left(E_{l}\right) \rightarrow h(E)}$ and $d_{l\left(E_{l}\right) \rightarrow h(E)}$ and negligible energy loss at high energies (no energy derivative), each equation is a homogeneous linear first order differential equation where the solutions have simple exponential forms. The couplings, either due to inelastic hadronic interactions of hadrons with air or due to the decay in hadronic or semi-leptonic final states, link the evolution of different species $h$ and thus increase subsequently the order of the system.

The coefficient of the interaction couplings consist of inclusive particle production spectra

$$
c_{l\left(E_{l}\right) \rightarrow h(E)}=\frac{1}{\sigma_{\text {inel }, l-\text { Air }}\left(E_{l}\right)} \frac{\mathrm{d} \sigma_{h}\left(E_{l}\right)}{\mathrm{d} x_{L}} \stackrel{x_{L}=\frac{E}{E_{l}}}{=} E_{l} \frac{\mathrm{d} N\left(E_{l}\right)}{\mathrm{d} E} \approx x_{F} \frac{\mathrm{d} N\left(E_{l}\right)}{\mathrm{d} x_{F}} .
$$




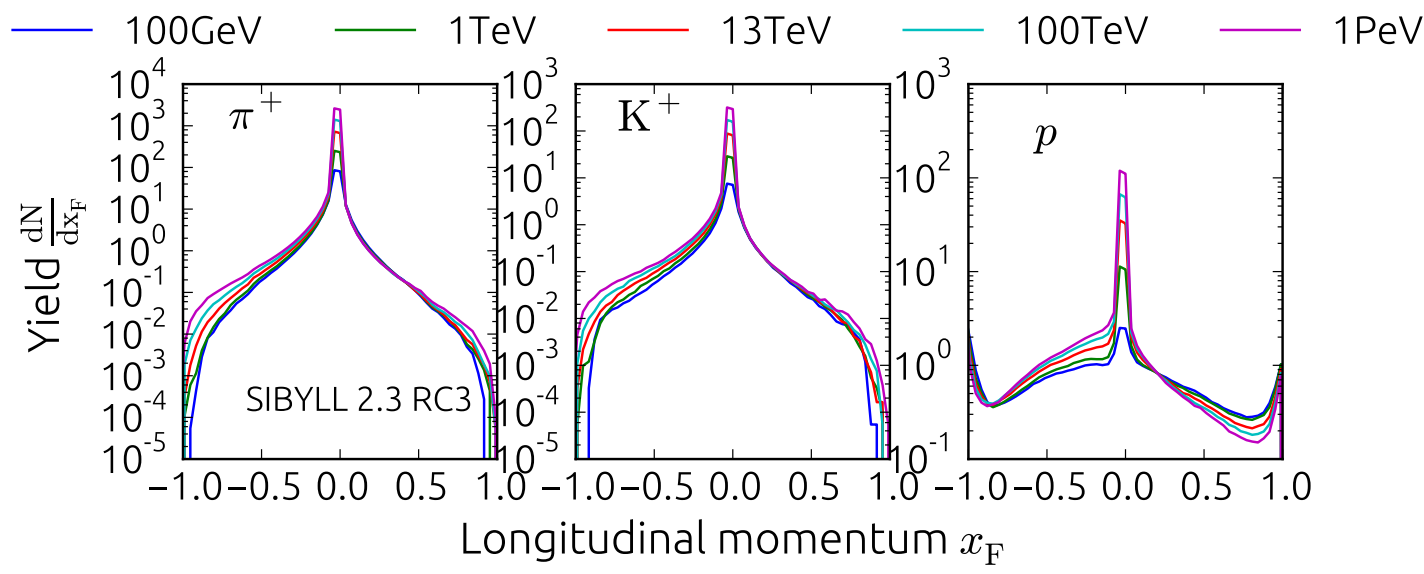

Figure 1: Feynman scaling of inclusive particle spectra in SIBYLL 2.3 RC3 in proton-air interactions.

This expression allows for observation of Feynman scaling in projectile fragmentation region. Figure 1, where particle production spectra are generated with SIBYLL 2.3 RC3, shows that pion and kaon spectra obey Feynman scaling at mid $x_{F}$, i.e. a universal form of the forward spectrum. In the central region $x_{F}<0.1$, the increasing number of multiple parton interactions enhances pair-production and violates scaling, as expected. At forward large $x_{F}$ scaling is violated for most particles in SIBYLL 2.3 RC3 since they are associated with the fragmentation of the projectile remnant. Proton spectra do not perfectly scale, since they can undergo additional processes, like diffraction, as a part of the projectile.

In typical semi-analytical solutions of the atmospheric lepton problem [1,2], the scaling argument is used to derive energy independent couplings factors, the spectrum weighted moments or Zfactors. This approach works for power-law cosmic ray spectra in the case where the solutions factorize $\Phi_{l}\left(E_{l}, X\right)=A_{l}(X) E_{l}^{-\gamma}$ and the interaction length is independent of energy $\lambda_{\text {int }, l}\left(E_{l}\right) \approx \lambda_{\text {int }, l}$. The definition follows from Eq. (1.2) and the RHS of Eq. (1.1)

$$
\begin{aligned}
A_{l}(X) \int_{E}^{\infty} d E_{l} E_{l}^{-\gamma} \frac{c_{l\left(E_{l}\right) \rightarrow h(E)}}{\lambda_{\text {int }, l}\left(E_{l}\right)} & =\frac{A_{l}(X)}{\lambda_{\text {int }, l}} \int_{E}^{\infty} d E_{l} E_{l}^{-\gamma+1} \frac{\mathrm{d} N_{h}\left(E_{l}\right)}{\mathrm{d} E} \\
& =\frac{A_{l}(X)}{\lambda_{\text {int }, l}} \int_{0}^{1} d x_{L} x_{L}^{-\gamma+1} \frac{\mathrm{d} N_{h}}{\mathrm{~d} x_{L}} \\
& =\frac{A_{l}(X)}{\lambda_{\text {int }, l}} Z_{l h .} .
\end{aligned}
$$

A more general approach, the energy dependent $Z$-factor, using piecewise power-law primary spectra has been introduced in [3]. If scaling holds in the phase-space region relevant for inclusive lepton fluxes, one expects constant spectrum-weighted moments a constant power-law index. Figure 2 shows the moments for a fixed cosmic ray spectral index $\gamma=2.7$. While $Z_{p \pi}$ is approximately constant, the larger correlation of kaons due to associated production with the strange forward baryons baryons, results in slightly growing $Z$-factors. Generally, the new version of SIBYLL obeys better scaling behaviour than the old SIBYLL 2.1. For our calculations the interaction coefficients are calculated directly using an interface to various Monte Carlo interaction models.

Particle decays are Lorentz-invariant. The interaction coefficients can therefore be directly 


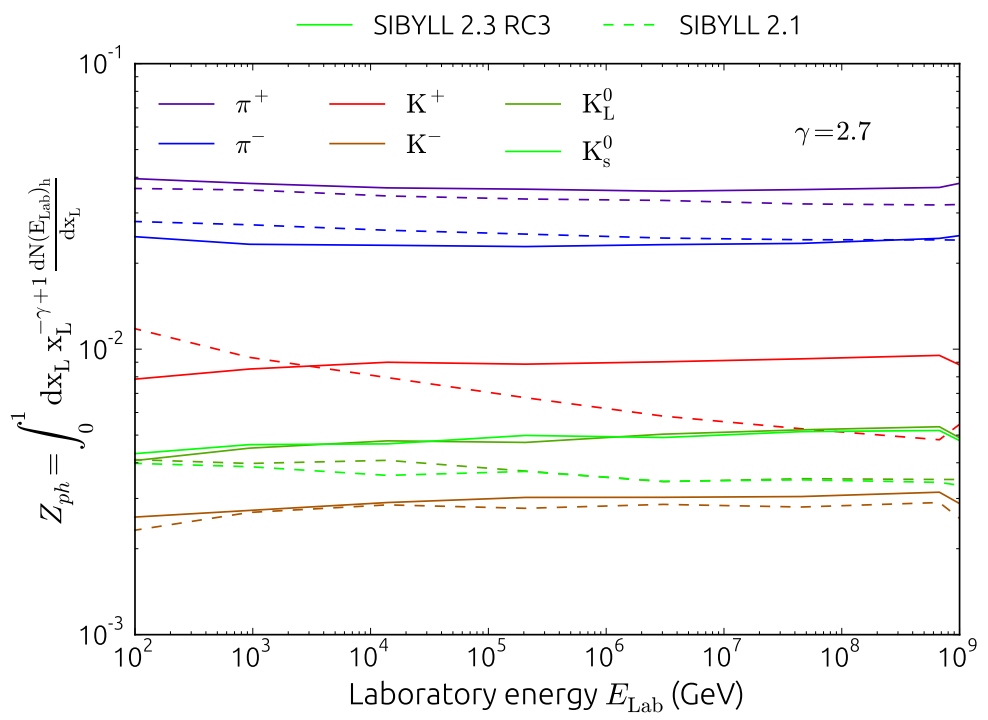

Figure 2: Spectrum-weighted moments for SIBYLL 2.1 and SIBYLL 2.3 RC3.

written as a function of $x_{L}$

$$
d_{l\left(E_{l}\right) \rightarrow h(E)}=B R(l \rightarrow h) \frac{\mathrm{d} N_{h}\left(E_{l}\right)}{\mathrm{d} E} \stackrel{x_{L}=\frac{E}{E_{l}}}{=} B R(l \rightarrow h) \frac{\mathrm{d} N_{h}}{\mathrm{~d} x_{L}} .
$$

At high energies rare decay channels with branching ratios $B R<10^{-4}$ have to be included in prompt flux calculations. The decay coefficients are obtained from the PYTHIA 8 Monte Carlo code [4].

For our numerical program, Matrix Cascade Equations (MCEQ), it is convenient to write the system of cascade equations as a matrix expression. The state vector is generated by writing the solutions $\Phi_{h}\left(E_{i}\right)$ for each hadron $h$ and discrete energy bin $E_{i}$ and into a column vector $\Phi$

$$
\Phi=\left(\begin{array}{c}
\Phi_{p}\left(E_{0}\right) \\
\Phi_{p}\left(E_{1}\right) \\
\cdots \\
\Phi_{p}\left(E_{N}\right) \\
\Phi_{n}\left(E_{0}\right) \\
\cdots
\end{array}\right) .
$$

The energy grid $E_{i}=50 \mathrm{GeV} \cdot 10^{i / N}$ is contains $\sim 8$ bins per decade of energy across the valid energy range between $50 \mathrm{GeV}$ and $10^{10} \mathrm{GeV}$. More than 60 types of mesons, baryons and leptons are taken into account. Together with some additional technical groups the dimension of $\Phi$ exceeds $\sim 6000$.

Using the definitions from [7] the coupled cascade equations in Eq. (1.1) are represented by this matrix equation

$$
\frac{\mathrm{d}}{\mathrm{d} X} \Phi=\left[(-1+C) \Lambda_{\text {int }}+\frac{1}{\rho(X)}(-1+D) \Lambda_{d e c}\right] \Phi
$$


Special care has to be taken when calculating prompt fluxes. Typically, the fastest mode restricts the maximum step-size in Runge-Kutta methods. The short life-time of heavy-flavour hadrons and resonances introduces very fast modes, making the system of equations stiff. As outlined in [7], the stiffness can be significantly reduced by neglecting re-interactions of short-lived particles with the atmosphere at lower energies.

\section{Inclusive muon fluxes and charge ratio}

An unfolded measurement of the inclusive muon flux and charge ratio was performed with very high precision by the L3 collaboration at CERN [8]. This data provides us a valuable crosscheck of the interaction model and the numerical approach. At energies around $100 \mathrm{GeV}$ the muon flux is most sensitive to the pion production at high $x_{F}$. At $\mathrm{TeV}$ energies the absorption of pions in the atmosphere increases the importance of the contribution of muons from charged kaon decay. The muon charge ratio is sensitive to the charge ratio of pion and kaon secondaries in hadronic interactions at $x_{F} \gtrsim 0.3$ and their relative abundance as mother particle of the leptons, sometimes referred to as $\pi / K$-ratio. Comparisons using development versions of SIBYLL 2.3 revealed that the models' performance with respect to low-energy fixed-target data from NA49 $[17,18,19]$ is strongly correlated with the fluxes, i.e. a lack of $\pi^{ \pm}$at $0.3<x_{F}<0.6$ resulted in a similar deficit in the muon flux. Equivalent behaviour can be observed regarding the connection between meson charge ratios and the muon charge ratio. Muon observations at high energies are therefore suitable to gain some detailed inference back into the interaction model development and extend the knowledge of the forward phase-space to higher energies. The fluxes in Figure 3 are calculated with MCEQ using a model of the atmosphere above Geneva from NRLMSISE-00 [20]. Assuming independence of the pion and kaon components of the muon flux, we performed an exercise in fitting fudge factors using the data from Figure 3. The prediction was slightly improved by rescaling normalization of the pion component by roughly $5 \%$ up and the kaon component by $+15 \%$ for $K^{+}$and $+40 \%$ for $K^{-}$. Since the majority of conventional neutrinos originates from $K^{ \pm}$and $K_{L}^{0}$ decays, the adjustment of the interaction model to the flux and charge ratio data simultaneously notably improves the predictive power of our calculations in the atmospheric neutrino sector.

The spherical geometry in the code and the numerical integration of arbitrary density profiles of the atmosphere provide access to detailed information about the formation of the flux as a function of the altitude or depth. In Figure 4 one can see the exponential forms of the $X$ dependent particle production in upper atmosphere and the decay dominated part of an inclined air-shower as linear height dependence at lower altitudes. Such calculations can be performed for all types of leptons or hadrons, giving users the possibility to study the dependence on the atmosphere in high detail.

\section{Prompt neutrinos from charm decays}

As a important background for astrophysical neutrinos at $\mathrm{PeV}$ energies, calculations of the prompt neutrino flux are one of the main features in MCEQ. Heavy flavour production is part of the default event generation scheme in SIBYLL 2.3. Details about contributing perturbative and non-perturbative processes are given in [5]. Predictions of the prompt flux are shown on the left 

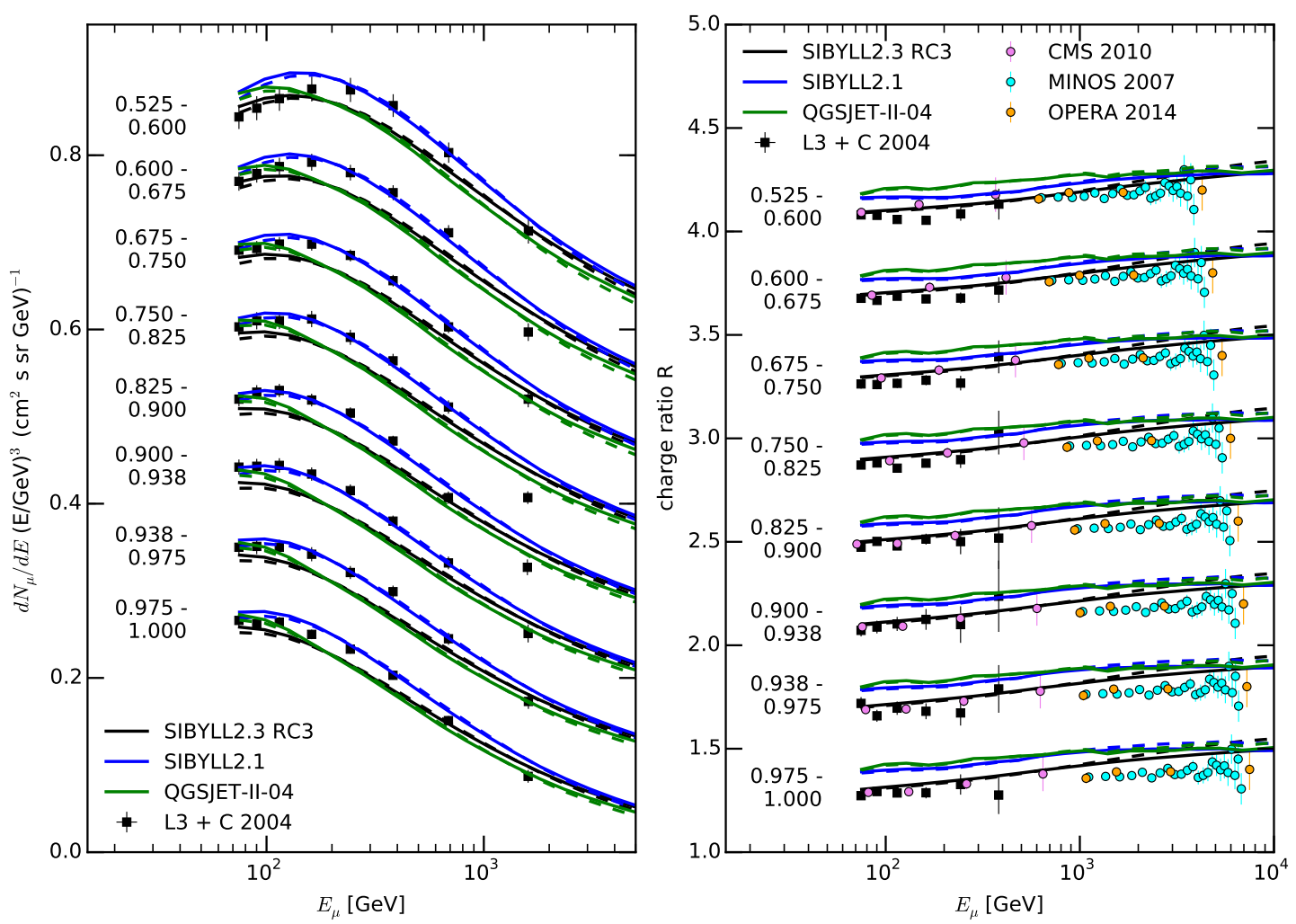

Figure 3: Inclusive muon flux and muon charge ratio compared to subset of available experimental data [8, 10, 11, 9]. Solid lines are computations using the H3a primary flux [12] and GST (3-generations) [13] for the dashed, respectively.

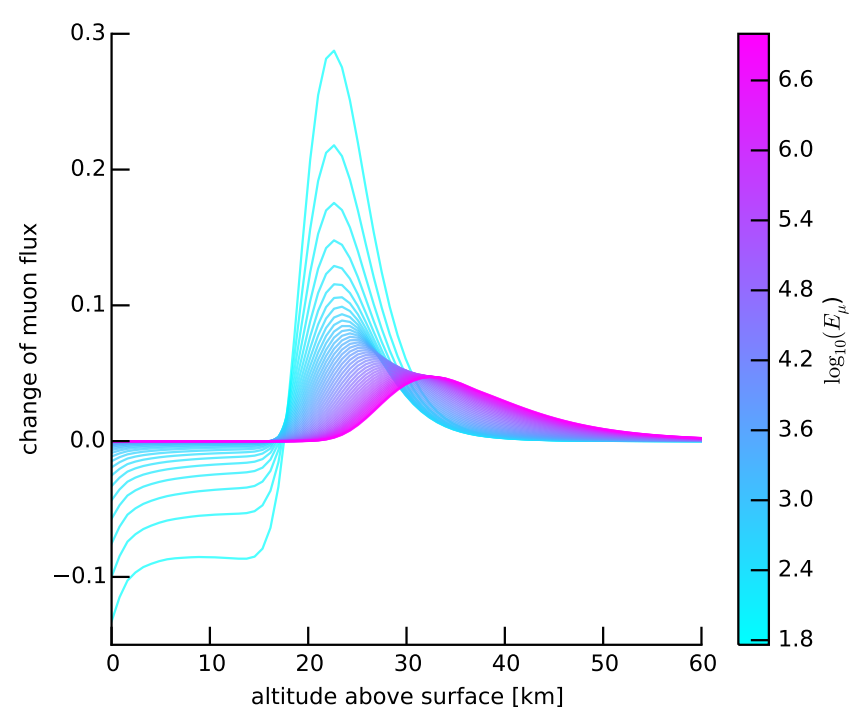

Figure 4: Derivative of muon flux for a very inclined shower from a proton primary at $10 \mathrm{PeV}$ and $\theta_{\text {zenith }}=$ $89^{\circ}$, plotted against the altitude above the surface under the trajectory of the shower. Each coloured line is a selection of muons in a small energy band. Negative values represent muon decay. 

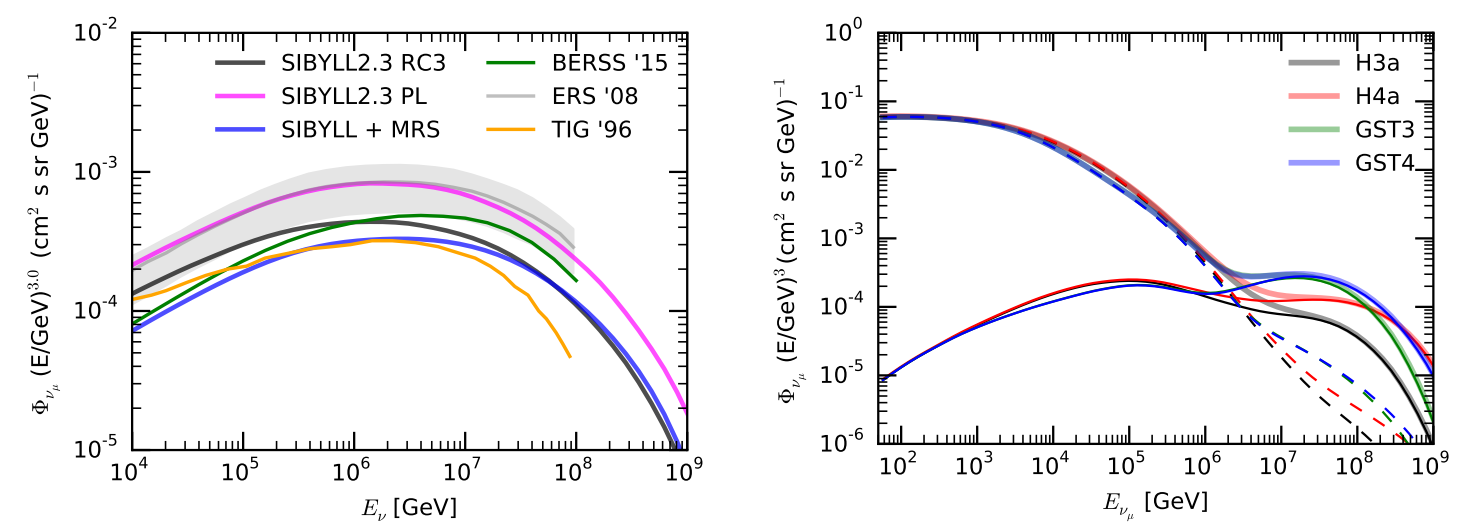

Figure 5: (left) Prompt muon neutrino flux calculated using SIBYLL 2.3 RC3 and a simple broken powerlaw model from TIG[3]. Superimposed are ERS [14], BERSS [15] and MRS [16]. (right) Dependence of the atmospheric muon neutrino flux on the primary cosmic ray spectrum and composition provided by more realistic models $[12,13]$.

panel of Figure 5. Changes to the baryon production model in the current RC3 version of SIBYLL result in a lower flux with respect to the $\mathrm{RC} 1$ version [5]. The uncertainty due to the nuclear model is investigated following the approach in [7], where the production of charmed quarks is approximated as a point-like (PL) process. The cross-section for charm production in air is obtained from $p p$ interactions by

$$
\sigma_{c \bar{c}, p-a i r}=A_{a i r} \sigma_{c \bar{c}, p-p}=14.5 \sigma_{c \bar{c}, p-p} .
$$

In MCEQ, any charm model can be interfaced with the solver by providing tables or approximate analytic expressions, as for example Eq. (29) in [16], for the differential $c \bar{c}$ or charmed meson cross-section $\mathrm{d} \sigma_{\text {charm }} / \mathrm{d} x$. In this case, the conventional hadronic cascade is calculated using any interaction model. An advantage of MCEQ is, that all of its features, like variation of atmosphere, primary flux and angular dependence are still available using external models.

In the right panel of Figure 5 the lighter composition above the ankle in the $H 4 a$ and both GST models $[12,13]$ yields a significantly higher neutrino flux above the (neutrino) knee. It is important to recognise that the uncertainty associated with the composition is of similar magnitude as the one associated with charm production.

\section{Outlook and conclusions}

MCEQ, as a generic solver of cascade equations, has been applied to calculate predictions of inclusive atmospheric lepton fluxes. The program contains a special method to deal with decays of short-lived particles which affect the stiffness of the equations and increases the calculation time. The, still in development, PYTHON program ${ }^{1}$ is open-source and it contains all ingredients for flux calculations. Planned features for the next versions include the extension towards lower energies, higher dimensional shower geometry and support for more general targets, in addition to air.

\footnotetext{
${ }^{1}$ https://github.com/afedynitch/MCEQ
} 
We demonstrated that at energies between tens of $\mathrm{GeV}$ and $\mathrm{TeV}$ our calculations reproduce the atmospheric muon flux with reasonable uncertainties. The remaining differences were traced back to deficiencies in the description of particle spectra in relevant phase-space by the interaction model. Using scaling arguments, available muon measurements give, as an inverse problem, insight into forward particle physics beyond energies of current fixed-target measurements. We show that the spectrum and composition of primary cosmic rays energies around and above the ankle, contribute at the same order of magnitude to total uncertainty of the flux above the lepton knee.

\section{Acknowledgment}

We are grateful to our colleagues from the IceCube Collaboration for their interest in this program and the many fruitful discussions. In particular Gabriel Collin who employed this code in his active research. This work is partly sponsored by the Wolfgang Gentner Programme of the Federal Ministry of Education and Research (BMBF) and the Helmholtz Alliance for Astroparticle Physics (HAP), which is funded by the Initiative and Networking Fund of the Helmholtz Association.

\section{References}

[1] T. K. Gaisser, Cambridge, UK: Univ. Pr. (1990) 279 p

[2] P. Lipari, Astropart. Phys. 1 (1993) 195.

[3] P. Gondolo, G. Ingelman and M. Thunman, Astropart. Phys. 5 (1996) 309 [hep-ph/9505417].

[4] T. Sjöstrand et al., Comput. Phys. Commun. 191 (2015) 159 [arXiv:1410.3012 [hep-ph]].

[5] F. Riehn, R. Engel, A. Fedynitch, T. K. Gaisser and T. Stanev, arXiv:1502.06353 [hep-ph].

[6] F. Riehn, R. Engel, A. Fedynitch, T. K. Gaisser, T. Stanev, This conference.

[7] A. Fedynitch, R. Engel, T. K. Gaisser, F. Riehn and T. Stanev, arXiv:1503.00544 [hep-ph].

[8] P. Achard et al. [L3 Collaboration], Phys. Lett. B 598 (2004) 15 [hep-ex/0408114].

[9] P. Adamson et al. [MINOS Collaboration], Phys. Rev. D 76 (2007) 052003

[10] N. Agafonova et al., Eur. Phys. J. C 74 (2014) 7, 2933.

[11] V. Khachatryan et al. [CMS Collaboration], Phys. Lett. B 692 (2010) 83 [arXiv:1005.5332 [hep-ex]].

[12] T. K. Gaisser, Astropart. Phys. 35 (2012) 801 [arXiv:1111.6675 [astro-ph.HE]].

[13] T. K. Gaisser, T. Stanev and S. Tilav, Front. Phys. China 8 (2013) 748 [arXiv:1303.3565 [astro-ph.HE]].

[14] R. Enberg, M. H. Reno and I. Sarcevic, Phys. Rev. D 78, 043005 (2008) [arXiv:0806.0418 [hep-ph]].

[15] A. Bhattacharya, R. Enberg, M. H. Reno, I. Sarcevic and A. Stasto, JHEP 1506 (2015) 110 [arXiv:1502.01076 [hep-ph]].

[16] A. D. Martin, M. G. Ryskin and A. M. Stasto, Acta Phys. Polon. B 34, 3273 (2003) [hep-ph/0302140].

[17] C. Alt et al. [NA49 Collaboration], Eur. Phys. J. C 45 (2006) 343 [hep-ex/0510009].

[18] C. Alt et al. [NA49 Collaboration], Eur. Phys. J. C 49 (2007) 897 [hep-ex/0606028].

[19] T. Anticic et al. [NA49 Collaboration], Eur. Phys. J. C 68 (2010) 1 [arXiv:1004.1889 [hep-ex]].

[20] Picone, J. M., A. E. Hedin, D. P. Drob, and A. C. Aikin, J. Geophys. Res., 107(A12), 1468, doi:10.1029/2002JA009430, 2002. 